\title{
PROPUESTA DE MODELO DE MEJORA PARA MYPES PRODUCTORAS DE SOFTWARE
}

\author{
Miriam Elizabeth Amable Ciudad \\ miriama@ulima.edu.pe \\ Universidad de Lima, Lima, Perú
}

\section{Resumen}

Este trabajo se basa en el resultado de la investigación "Análisis del uso de modelos de calidad de software. Una propuesta de mejora de procesos en las mypes productoras de software de Lima", que contó con el apoyo del Instituto de Investigación Científica de la Universidad de Lima. El estudio caracterizó a las mypes y se demostró que no tienen un uso adecuado de los modelos de calidad de software, tanto en sus procesos como en sus productos. Además, estas empresas reconocieron que tenían problemas en sus procesos y necesitaban mejorarlos. Así, se planteó desarrollar otra investigación que propuso un modelo de mejora de procesos adecuado y sostenible a cada categoría de mype, basado en un análisis profundo de los modelos de calidad existentes, que pueden ser aplicables a todo el ciclo de vida del software: desde el establecimiento de requisitos hasta las pruebas de calidad.

Palabras clave: aseguramiento de calidad / mejora de procesos / mypes productoras de software

Abstract

\section{Proposed model improvement for "mypes" software producers}

This work is based on the results of the research "Analysis of the use of software quality models: a proposal to improve processes in the 'mypes' producing software in Lima", which was supported by the Institute of Scientific Research of the University of Lima. The study characterized "mypes" and showed that they do not have an adequate use of software quality models, both in their processes and products. In addition, these companies recognized that they have problems in their processes and the need to improve them. It was therefore proposed to develop a further research setting a suitable and sustainable process improvement model for each category of "mype", based on an in-depth analysis of existing quality models, that could be applied throughout the entire software lifecycle: from the establishment of requirements to the quality tests.

Keywords: Quality assurance / process improvement / software producing mypes 


\section{Introducción}

El presente artículo expone el desarrollo de un modelo de mejora de procesos, adecuado y sostenible a cada categoría de mype, y basado en un análisis profundo de los modelos de calidad existentes, aplicables a todo el ciclo de vida del software.

Una primera etapa es categorizar las mypes productoras de software, a través de la tipificación de las empresas por el tipo de cliente que atienden, utilizándose la técnica de análisis de la organización para segmentarlas. La segunda etapa consiste en analizar los modelos de calidad existentes para la mejora de procesos; mediante la revisión sistemática de la literatura sobre estos modelos en micro, pequeñas y medianas empresas desarrolladoras de software. En la tercera etapa se elige el modelo de mejora de procesos adecuado a la categoría de mype, estableciendo la estrategia de implementación que incluye el entrenamiento en modelo. Finalmente, la cuarta etapa consiste en validar el modelo de mejora.

En la presente propuesta, se determinó la disponibilidad de recursos por parte de las mypes productoras de software, la disposición de implementar un modelo de mejora y sus opiniones al respecto, la validación cuantitativa ha quedado para estudios posteriores.

\section{Problema de la investigación}

Sobre la caracterización de las mypes productoras de software de Lima se registraron los siguientes hallazgos:

Un 66,3\% de las empresas encuestadas no consideraba la utilización de los modelos de calidad de proceso más conocidos en el mercado. Esto se demostraba en el resultado, en el cual se observaba el bajo uso y conocimiento de los estándares de calidad en las mypes. (Amable, 2015, p. 90)

Y en relación con los modelos orientados al producto, Amable (2015) precisa:

El 27,6 \% de las empresas aplicaba estándares de calidad de producto software; $\sin$ embargo, había un $42,5 \%$ que empleaba sus propios métodos y un $38,8 \%$ que no utilizaba ningún modelo. Se concluyó que muy pocas empresas conocían los estándares de calidad de producto y por tal razón no los aplicaban. (p. 90)

Sobre la base de la situación presentada se determinó que era necesario establecer modelos de calidad en las mypes productoras de software, planteándose la siguiente pregunta de investigación para el presente estudio: ¿Cómo un modelo de mejora de procesos adecuado ayudaría al mejor desempeño de las mypes productoras de software? 


\section{Estado del arte}

En Amable, Millones y Checa (2015), se expone que la mayoría de mypes de Lima metropolitana tienden a realizar sus labores mediante el uso de empirismos aplicativos y organizativos, pero también son conscientes de la necesidad de crear productos software con calidad que cumplan con las expectativas de los clientes, y así alcanzar una ventaja competitiva. Por ello, las autoras mencionadas indican que los beneficiarios de la investigación realizada eran las mypes productoras de software así como los investigadores, quienes tendrían la oportunidad de realizar propuestas que contribuyan a mejorar los procesos de desarrollo de estas organizaciones, utilizando modelos de calidad de software adecuados.

Osterwalder y Pigneur (2011) indican que hay que caracterizar la organización mediante el modelo de negocio, el cual es definido como el modelo que "describe las bases sobre las que una empresa crea, proporciona y capta valor" (p. 14). Así también, Barrios (2010) define como modelo de negocio al conjunto complejo de rutinas interdependientes que se descubren, ajustan y matizan mediante la acción. El término más importante dentro de esta definición está dado por la palabra interdependientes, y esta es una de las principales diferencias de esta herramienta. Se suele definir como un negocio o empresa a la generación de valor a través de la utilización de la cadena de valor. En relación con la propuesta de valor, según los ejemplos brindados por Osterwalder y Pigneur (2011) en las organizaciones productoras de software se puede considerar como el modelo basado en la personalización, el cual se indica que crea valor, ya que el cliente de productos software exige calidad y adaptabilidad a sus necesidades. Otro modelo propuesto en Osterwalder y Pigneur (2011) es el basado en diseño, que es "un factor importante, aunque difícil de medir. Un producto puede destacar por la superior calidad de su diseño" (p. 24). Esto es muy importante, ya que se sigue un ciclo de vida del desarrollo de software para elaborar el producto software, y para asegurar la calidad es necesario realizar su medición; por ello, los proyectos de mejora de procesos deben incluir un modelo de medición para asegurar la calidad y el éxito de la aplicación de los modelos de mejora de procesos. Esto se observa también en García, Mitre, De Amescua y Velasco (2010), que muestran un modelo de medición basado en que, desde la perspectiva de la gestión estratégica, se debe lograr que las organizaciones cumplan sus objetivos competitivos; y al aplicar una mejora de procesos de software, esta debe alinearse con los objetivos estratégicos; asimismo, para saber que esto se cumple debe medirse. En dicho artículo se propone el modelo de medición: Método Balanceado Objetivo-Cuantificadores (Balanced Objective-Quantifiers Methodology - BOQM) el cual integra la gestión estratégica, la mejora de procesos y la medición cuantitativa para lograr la competitividad de las organizaciones de ingeniería de software.

Además, en García et al. (2010) se indica que son pocas las iniciativas de mejora de procesos de software que se han alineado a la estrategia de la organización, porque no incorporaron mediciones cuantitativas controladas por un programa de medición. Es importante destacar la hipótesis planteada en García et al. (2010), donde se indica que: 
si hay una metodología disponible, especialmente dirigida a Organizaciones de Ingeniería de Software (OIS), que integran actividades de gestión estratégica, principios de mejora de procesos y técnicas de medición, será posible dirigir una estrategia de OIS, potencialmente incrementando las sinergias entre todos los interesados (stakeholders) y distribuyendo el conocimientos en todos los niveles para una toma de decisiones efectiva. (p. 342)

También en Mitre, De Amescua, García y Velasco (2014) se indica que es importante asegurar la calidad de un producto software, considerando que esa tarea es difícil para las organizaciones; sin embargo, es importante diseñar un eficiente programa de medición estratégico, sobre todo cuando se aplican proyectos de mejora de procesos de software. Además, presenta un estudio piloto para observar y analizar la operación de equipos de medición utilizando tres métodos de medición: el método balanceado objetivo-cuantificadores - BOQM, el método de medición práctica del software (Practical Software Measurement - PSM) y el método integrado cuadro de mando integral y medición dirigido a las metas (Balanced Scorecard and Goal-Driven Measurement - BSC\&GQ[i]M). En ese estudio se muestran las dificultades para diseñar el programa, alinearlos a las metas estratégicas y justificar las utilidades o beneficios de integrar las iniciativas de mejora con la estrategia del negocio.

Otro aspecto fundamental en la industria del software es el módulo de canales, considerado en Osterwalder y Pigneur (2011) como "el modo en que una empresa se comunica con los diferentes segmentos de mercado para llegar a ellos y proporcionarles una propuesta de valor" (p. 26). Esto es esencial en el aseguramiento de calidad, ya que debe satisfacer la expectativa de los clientes y medirla. Para ello, debe existir una adecuada y efectiva comunicación con los clientes, y considerarse sus distintos tipos de estos; por ejemplo, ser proveedor de un cliente de una organización del Estado es distinto a ser proveedor de un cliente de una organización privada, aunque en este último caso también se podría diferenciar por tipo de producto. Asimismo, en el caso de clientes nacionales y clientes extranjeros, hay una diferencia respecto de los requisitos que se deben cumplir; entonces, los canales de comunicación, distribución y venta deben ser identificados y gestionados de una manera efectiva. En Osterwalder y Pigneur (2011) se presentan preguntas a este respecto:

¿Qué canales prefieren nuestros segmentos de mercado? ¿Cómo establecemos actualmente el contacto con los clientes? ¿Cómo se conjugan nuestros canales? ¿Cuáles tienen mejores resultados? ¿Cuáles son más rentables? ¿Cómo se integran en las actividades diarias de los clientes? (p. 27)

Respecto al desarrollo de software, se debe considerar el aseguramiento de calidad de software, que en Moreno, Sánchez, Colomo y De Amescua (2014) se presenta como un trabajo realizado a empresas de desarrollo de software en Corea del Sur, el cual reveló que casi la mitad de las compañías no usaban un plan de aseguramiento de calidad de software 
en sus proyectos. Del mismo modo, como las debilidades de las empresas del sector existían, la situación de dichas organizaciones requería mejorar. La encuesta fue realizada a 34 empresas de tres tamaños: pequeña, mediana y gran empresa. Esto se refuerza con lo señalado en Amable et al. (2015), a través del estudio cuantitativo de una muestra representativa, donde se identificaron estas situaciones: el $66,3 \%$ no utilizaba los modelos de calidad de procesos CMMI, Moprosoft, ISO/IEC 12207 o ISO/IEC 29110, por desconocimiento de estos; sin embargo, el $48 \%$ de las empresas manifestaron que deseaban certificarse en algún modelo, debido a que tenían la oportunidad de exportar. Por otro lado, el 81,9 \% desconocía los modelos de calidad de producto McCall, ISO/IEC 9126 e ISO/IEC 25000; sin embargo, sí reconocían el uso de factores de calidad de software. El estudio se realizó siguiendo el proceso de investigación cuantitativa, como se detalla en Amable (2015).

En Garzás, Pino, Piattini y Fernández (2013) se resalta que, partiendo de la necesidad de muchas organizaciones en implementar modelos de mejora de procesos para elevar su madurez organizacional en el desarrollo de software, existen modelos tradicionales de madurez que implican una gran inversión que no puede ser asumida por las organizaciones pequeñas; entonces, es necesario considerar un modelo adecuado para tales organizaciones. Asimismo, en Niazi, Babar y Verner (2010), se indica que en las iniciativas de mejora de procesos de software se identifican barreras que pueden perjudicar la aplicación de un modelo de mejora de procesos. Tales barreras son: (1) falta de gestión de proyectos, (2) falta de recursos, (3) falta de patrocinio, (4) personal sin experiencia / falta de conocimiento, y (5) falta de conciencia en la mejora de procesos.

Para proponer un modelo de mejora de procesos es preciso realizar una evaluación; por eso, en Von Wangenheim, C., Rossa, Salviano y Von Wangenheim, A. (2010) se menciona que la evaluación y mejora de procesos de software puede ser efectuada a través de un perfil de nivel de madurez o capacidad de proceso basado en un modelo adecuado. Para poder identificar el modelo adecuado para una organización es necesario llevar a cabo una investigación, tal como una revisión de literatura sistemática. En Kitchenham y Charters (2007) se indica que la mayoría de las investigaciones se inician con una revisión de la literatura de algún tipo, sin embargo, a menos que sea exhaustiva, es de poco valor científico. Esta es la principal razón para la realización de revisiones sistemáticas. Una revisión sistemática sintetiza el trabajo existente de una manera objetiva. Para ello es preciso seguir una estrategia de búsqueda predefinida, la cual es formada por la pregunta de investigación, descriptores temáticos relevantes y tiempo en que se realiza la investigación.

En Zarour, Abran, Desharnais y Alarifi (2015) se sigue las etapas de la revisión sistemática de la literatura y se tiene como objetivo presentar los resultados de la literatura sobre 38 mejores prácticas de la Evaluación de Procesos de Software (EPS). Tales prácticas se presentan en la literatura como requisitos de evaluación, factores de éxito, observaciones y lecciones aprendidas. Se han clasificado en cinco categorías principales: las prácticas relacionadas con los 
métodos EPS, las herramientas de apoyo, los procedimientos, la documentación y los usuarios. Lo que se propone en este artículo es que se diseñen métodos de evaluación ligeros adaptados a la experiencia individual.

En Moreno et al. (2014) se expone que la industria de software reconoce la contribución que dan las pequeñas organizaciones en los productos y servicios que brindan. Asimismo, se menciona que las normas ISO/IEC no estaban dirigidas a las pequeñas organizaciones hasta que se desarrolló la norma ISO/IEC 29110. Como resultado de la creación de esta norma, se han planteado modelos de evaluación y mejora de procesos de software, paquetes de despliegue, proyectos piloto y estrategias de implementación como apoyo a la norma, considerando herramientas de documentación y gestión del conocimiento para apoyar la adopción de la norma. En este estudio se busca establecer un modelo de mejora de procesos en el que se estudiará en profundidad la norma ISO/IEC 29110 entre otras normas y modelos de calidad. También se considerará la selección y propuesta de un conjunto de herramientas, tal como se indica en Moreno et al. (2014), que presenta un trabajo realizado a cuatro pequeñas empresas de desarrollo de software en España. Estas empresas se agruparon en un programa de mejora de proceso conjunto para la implementación de la norma ISO/IEC 29110, en el que se seleccionaron y propusieron un conjunto de herramientas de apoyo a algunas de las buenas prácticas de la Guía PMBOK en el entorno integrado de la gestión de proyectos de desarrollo de software con relación al alcance, tiempo y comunicaciones.

\section{Procedimiento y resultados}

\subsection{Etapa 1. Categorizar las mypes productoras de software}

Se considera la población delimitada en la investigación referida de Amable et al. (2015), identificándose que el $68 \%$ son microempresas productoras de software y el $32 \%$ son pequeñas empresas. En la tipificación de las empresas, un aspecto importante para poder identificar objetivos es el tipo de clientes que atienden. En las microempresas hay un 64,8 $\%$ que exportan productos software, representado por la cantidad de clientes extranjeros, y en la pequeña empresa hay un 69,2 \% de clientes extranjeros. Esta concentración de clientes extranjeros exige un proceso de desarrollo de software con calidad y sostenible en el tiempo para poder atender las exigencias de las organizaciones foráneas. Una de las exigencias es la certificación de los procesos de la organización, a través de un modelo de calidad que garantice su adecuada ejecución.

\subsection{Etapa 2. Analizar modelos de calidad de mejora de procesos}

En la etapa de la revisión sistemática se mostraron los resultados de la investigación que se ha efectuado y logrado sobre la base de los artículos encontrados respecto de la búsqueda avanzada, realizada en el tema de modelos de calidad de mejora de procesos 
en micro, pequeñas y medianas empresas desarrolladoras de software, en el formato de búsqueda realizada en las bases de datos consultadas, y en la comparación entre los modelos de calidad con los diferentes artículos encontrados.

La pregunta planteada para la revisión sistemática fue “¿Cuáles son las iniciativas que se han realizado para modelos de calidad en mejora de procesos y que al mismo tiempo presentan un caso real de estudio?".

En la revisión sistemática se considera la terminología, método de búsqueda, extracción de datos, análisis de los resultados y conclusiones.

Se buscó en las bases de datos Science Direct, IEEE XPLORE, EBSCOhost y PROQUEST. En ScienCEDirect se siguió el patrón de búsqueda (abstract, title, keywords: software process model and abstract, title, keywords: capability maturity) and (Moprosoft or Competisoft or ISO/IEC 29110) and (software process engineering CMM ISO MOPROSOFT) and (CMM or CMMI or ISO or Moprosoft).

En IEEE XPLORE se siguió el patrón de búsqueda (ABSTRACT: Process) and (ABSTRACT: Maturity) and (ABSTRACT: Model) AND (standard <or> model <or > framework) <and> ("software process" <or > "software engineering") <and > (assessment <or > improvement <or> capability <or> maturity) <and> (CMMI <or> 15504 <or> 12207 <or> 29110 <or> CMM <or > SPICE <or> MOPROSOFT <or> COMPETISOFT <or > iso <or standards)) AND (MOPROSOFT or COMPETISOFT or ISO/IEC 29110)

En EBSCOhost se siguió el patrón de búsqueda software process improvement capability maturity AND (MOPROSOFT or COMPETISOFT or ISO/IEC 29110)

En ProQuest se siguió el patrón de búsqueda ("software process" <or> "software processes" <or> "software engineering") <and> (assessment <or> improvement <or > capability <or> maturity) <and> (CMMI <or> $15504<0 r>12207<$ < r > "MPS.BR" <or> CMM <or $>$ SPICE <or > iso <or > standards) AND (model) <and > "software process" <or > "software engineering") <and> (assessment <or> improvement <or> capability <or> maturity) <and > (CMMI <or> $15504<$ or> 12207 <or> CMM <or> SPICE <or> iso <or> standards) AND (12207 <or > CMM <or > SPICE <or > iso <or > standards) AND (MOPROSOFT or COMPETISOFT or ISO/IEC 29110). 
En la figura 1 se muestra el resultado de la revisión sistemática.

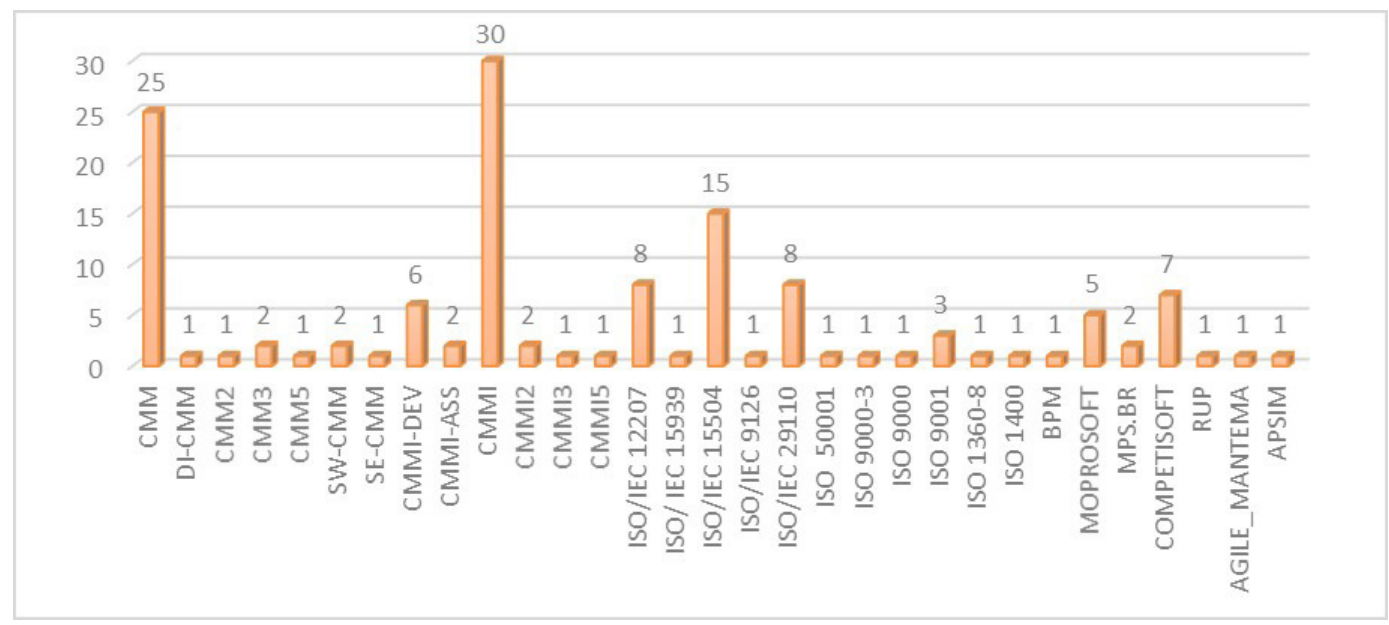

Elaboración propia

\subsection{Etapa 3. Determinar el modelo de mejora de procesos adecuado a categoría de mype}

Para esta etapa se identificaron las necesidades de cambio, las cuales reflejan la problemática que atraviesan las mypes productoras de software en relación con la producción de software. Para ello se realizaron entrevistas a seis organizaciones: cuatro microempresas y dos pequeñas empresas, obteniéndose los siguientes resultados relacionados con la ocurrencia de diversos problemas en las organizaciones participantes:

i. Reclamos de clientes: el $100 \%$ de las microempresas indicó que era algo frecuente, mientras que en la pequeña empresa el 50 \% señaló que tanto era nada frecuente como algo frecuente.

ii. Defectos en el producto software: los problemas de calidad del producto software eran frecuentes en el $75 \%$ y $50 \%$ de las micro y pequeñas empresas, respectivamente.

iii. Costos elevados: eran frecuentes en el $50 \%$ tanto en las micro como en las pequeñas empresas. En el $25 \%$ de las microempresas era muy frecuente.

iv. Recursos limitados: tanto las microempresas como las pequeñas empresas indicaron que ocurría entre frecuente y muy frecuente, en $50 \%$ para cada caso.

v. Incumplimiento de plazos de entrega: tanto las microempresas como las pequeñas empresas señalaron que ocurría como algo frecuente y frecuente en un $50 \%$. 
vi. Baja productividad: el $25 \%$ de las microempresas y el $50 \%$ de las pequeñas empresas lo señalan como nada frecuente; mientras que el $25 \%$ en algo frecuente, frecuente y muy frecuente para la microempresa; y para la pequeña empresa el $50 \%$ indica que es algo frecuente.

Asimismo, se identificaron las áreas de mejora vinculadas con los procesos del ciclo de vida y los de apoyo. Los resultados obtenidos fueron:

i. Con relación al primer proceso, correspondiente a la primera fase del ciclo de vida, está la Administración de Requisitos. Para el 75 \% de las microempresas es un proceso que requiere mejora.

ii. Con relación al segundo proceso cuestionado, correspondiente a la fase del ciclo de vida Arquitectura y Diseño de Software, para el $75 \%$ de las microempresas es un proceso que requiere mejora.

iii. Con relación al tercer proceso cuestionado, correspondiente a la fase del ciclo de vida Construcción de Software, para el 50 \% de las microempresas y pequeñas empresas es un proceso que requiere mejora.

iv. Con relación al cuarto proceso cuestionado, correspondiente a la fase del ciclo de vida Integración y Pruebas de Software, para el $75 \%$ de las microempresas y el $50 \%$ de pequeñas empresas es un proceso que requiere mejora. Esta situación se da por la percepción obtenida en el resultado de las pruebas de software, que mide la calidad de este.

v. Con relación al quinto proceso cuestionado, correspondiente a uno de los procesos de apoyo, Gestión de Proyectos, el $100 \%$ de las microempresas y el $50 \%$ de pequeñas empresas indican que es un proceso que requiere mejora.

vi. Con relación al sexto proceso cuestionado, correspondiente a uno de los procesos de apoyo, Gestión Financiera; solo el $25 \%$ de las microempresas indica que es un proceso que requiere mejora.

vii. Con relación al sétimo proceso cuestionado, correspondiente a uno de los procesos de apoyo, Mercadeo, el $25 \%$ de las microempresas y el $50 \%$ de las pequeñas empresas señalan que es un proceso que requiere mejora.

viii. Con relación al octavo proceso cuestionado, correspondiente a uno de los procesos de apoyo, Gestión de Recursos Humanos, solo el 25 \% de las microempresas señala que es un proceso que requiere mejora. 


\subsubsection{Modelo de mejora adecuado}

Un modelo de mejora adecuado debe ser aplicable en tres aspectos: calidad, costo y tiempo. No se trata de aplicar solo el modelo o norma de procesos, sino también el cómo, vinculado al método que se use en el desarrollo del software. En Amable (2015), de acuerdo a la figura 2, se mostraba que el 48,8 \% utilizaba metodologías de tipo prescriptivo, como RUP, y el 45,5\% utilizaba métodos ágiles de tipo adaptativo, principalmente SCRUM.

Figura 2. Metodologías / Prácticas de desarrollo de software

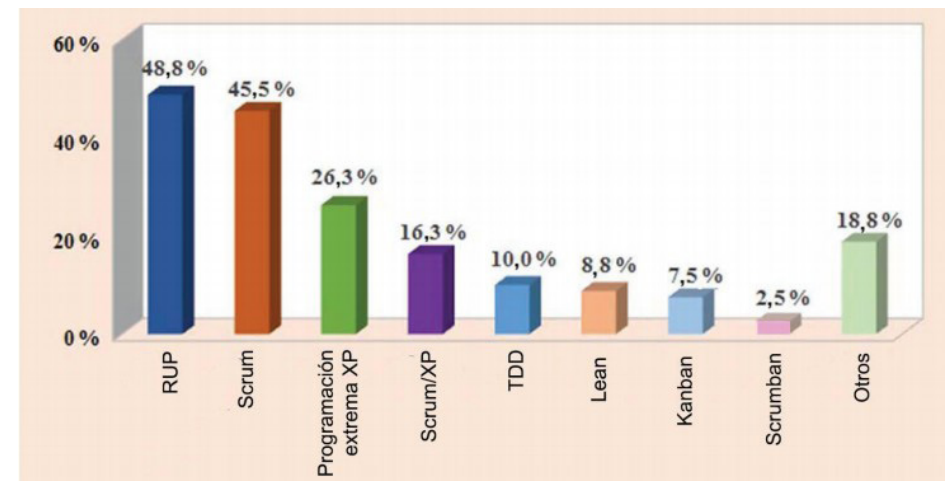

Fuente: Amable (2015)

En Galván, Mora, O'Connor, Acosta y Álvarez (2015) se estudió el cumplimiento de los métodos ágiles de desarrollo de software (SCRUM, XP) y la nueva norma ISO/ IEC 29110, debido a que el objetivo de ambos, tanto los métodos ágiles como la norma 29110, se dirigen a realizaciones sencillas. La propuesta de mejora de procesos significa seguir las indicaciones del modelo de referencia de la norma ISO/IEC 291105-1-2, del perfil básico, como modelo de referencia de procesos, combinado con alguna práctica metodológica que plantee el detalle del cómo lograrlo. Se considera una combinación con algún método ágil como el SCRUM, el cual era usado por la mayoría de las mypes. El proceso de modelo de mejora incluye una primera etapa que corresponde a la evaluación del proceso, y se utiliza el esquema mostrado en ISO / IEC. (2011), figura 3, correspondiente a la parte 3 de la norma ISO/IEC 29110. 
Figura 3. Elementos de la evaluación de proceso de la PO

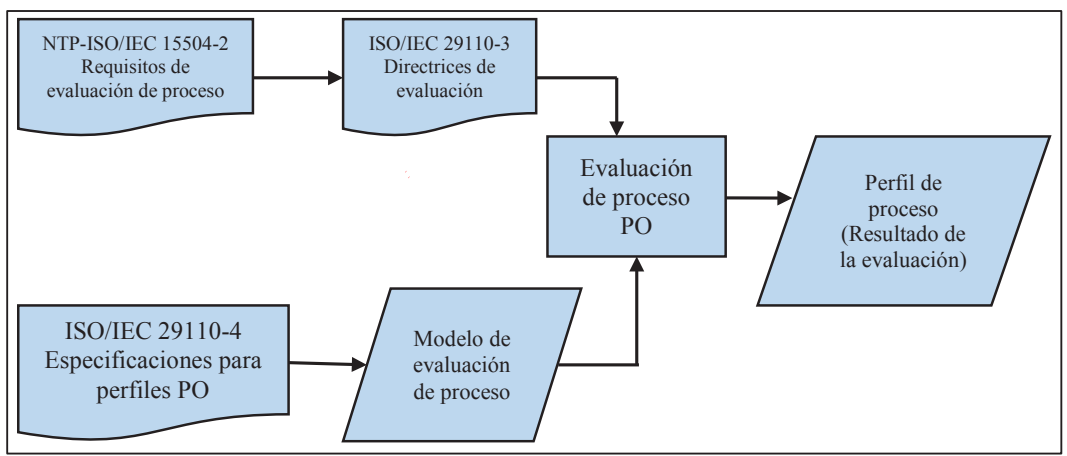

Fuente: ISO / IEC (2011)

Para una evaluación continua se propone que la organización realice autoevaluaciones periódicas, usando la herramienta de evaluación considerando el perfil básico de la pequeña organización propuesto en la norma ISO/IEC 29110, parte 5-1-2. En las actividades por mejorar se deben implementar las mejores prácticas planteadas para el desarrollo de software, principalmente los métodos ágiles. En el ciclo de mejora de procesos es importante evaluar continuamente el cumplimiento de la mejora de las actividades.

\subsection{Etapa 4. Validar el modelo de mejora con categoría de mype}

Como se señaló, el presente artículo muestra los aspectos asociados a una validación cualitativa, previa a una evaluación cuantitativa del modelo de mejora propuesto. Esta validación consiste en la determinación de la disponibilidad de los recursos para la mencionada implementación, la disposición de las mypes en implementar dicho modelo, así como las opiniones relacionadas a la importancia de los diversos aspectos del modelo de mejora. Con la explicación dada en un taller sobre el modelo de calidad propuesto, en este caso una combinación de la norma ISO/IEC 29110 y métodos ágiles del desarrollo de software, se determinó lo siguiente sobre la base de seis empresas: cuatro microempresas y dos pequeñas empresas:

\subsubsection{Disponibilidad de recursos}

i. Disposición de recursos: el 50 \% de las microempresas indicó que sí disponía de los recursos, y el 25 \% que no los tenía. En el caso de las pequeñas empresas, todas disponen de recursos para poder implantar un modelo de mejora.

ii. Existencia de un área independiente del desarrollo y mantenimiento de software para que sea responsable o lidere el proyecto de mejora de procesos (área de 
aseguramiento de calidad): solo una microempresa, que representa al $25 \%$ de las microempresas participantes, señaló que disponía de un área con esa funcionalidad, mientras que en el caso de las dos pequeñas empresas estas sí contaban con dichas áreas; cabe mencionar que ambas comenzaron como microempresas.

\subsubsection{Disposición favorable a la implementación}

i. Disposición de la organización para aplicar un modelo de mejora de procesos: el $100 \%$ de las microempresas y pequeñas empresas mostraron su disposición a implementar un modelo de mejora de procesos en su organización.

ii. Disposición para contratar una empresa especialista en el modelo de mejora: el $50 \%$ de las microempresas y el $100 \%$ de las pequeñas empresas respondieron afirmativamente.

iii. Disposición para realizar una autoevaluación en el modelo de referencia de procesos: el $100 \%$ de las microempresas estaría dispuesta a realizar una autoevaluación, mientras que solo un 50 \% de las pequeñas empresas lo haría. Cabe mencionar que se explicó que se deberá realizar una autoevaluación por cada ciclo de mejora de procesos ejecutado. Así se asegura la sostenibilidad en el tiempo a través de la mejora continua.

\subsubsection{Importancia de los aspectos del modelo de mejora}

i. Determinación de la misión, visión y objetivos de la empresa: el $100 \%$ de las microempresas la consideran entre importante y muy importante, y el $50 \%$ de las pequeñas empresas la considera muy importante. En Wasson (2015) se indica que para definir un modelo operacional es preciso tener claramente definida la misión y visión de la organización. Asimismo, no se puede emprender la construcción de software si antes no se ha definido un modelo operacional.

ii. Definición del alcance del proyecto: las respuestas obtenidas fueron de muy importante en un $75 \%$ para las microempresas, y en un $50 \%$ para las pequeñas empresas. Para iniciar un proyecto de mejora de procesos es preciso planificarlo. Un factor crítico de éxito es definir claramente el alcance de este, el cual debe ser establecido por la misma organización. Si se reconoce, en el resultado de la evaluación, que no cumple o cumple parcialmente, es necesario plantear una mejora para esa situación.

iii. Establecimiento de objetivos y metas: todos consideraron que es importante y muy importante realizar tal tarea; sobre todo cuando se desea implementar un proyecto de mejora de procesos. 
iv. Seguimiento sistemático de la mejora de procesos: la totalidad de las mypes respondió que es importante adoptar un modelo sistemático de mejora de procesos.

v. Monitoreo del proceso de cambio mediante un sistema de indicadores: tanto las microempresas como las pequeñas empresas consideran la importancia de definir un sistema de indicadores. Tal como se indica en Trujillo, Febles y León (2014), es adecuado aplicar indicadores y métricas para establecer la forma como realizar la valoración y orientar acciones que permitan desarrollar los aspectos positivos y contrarrestar los negativos.

vi. Adopción de un método ágil: el $75 \%$ de las microempresas y el $100 \%$ de las pequeñas empresas consideran importante y muy importante dicha adopción.

vii. Análisis de riesgos para identificar los cambios adecuados que permitan una sostenibilidad en el tiempo: tanto para las microempresas como para las pequeñas empresas es importante realizar un análisis de riesgos.

\subsubsection{Consideraciones adicionales}

En los comentarios vertidos durante el taller por los representantes de las empresas, tres de estas consideraron la exportación de software como un aspecto importante, y para lograrlo manifestaron que es necesario contar con una certificación en la norma indicada, expresando que:

siendo el cliente el actor principal de nuestro negocio, y conforme a nuestro deseo de exportar software (...) la única forma de alcanzar esa exportación es mediante la adopción de una certificación, porque el cliente conoce la certificación; por ello, si la tenemos ya nos conoce.

Teniendo en cuenta que la certificación de la norma se logra al demostrar que los procesos cumplen los requisitos de la norma, esto se consigue a través de proyectos de mejora de procesos; además, continuar con el manejo actual de los procesos significa una pérdida de dinero, tal como lo manifestó uno de los entrevistados:

Para una empresa lo más importante es entrar a la mejora de procesos, porque es muy posible que se esté perdiendo dinero por no tener los procesos adecuadamente, y esto no nos permite tener un crecimiento adecuado.

Otro entrevistado agregó:

Creo que todos coincidimos que es importante la mejora de procesos. Un modelo mal hecho es pérdida de dinero, el proyecto se extiende y se tiene al cliente descontento. Por eso lado es necesario mejorar nuestros procesos y lograr la calidad del entregable. 
Entonces es importante realizar una mejora de procesos si es que deseamos crecer; como lo manifestó otro entrevistado:

Si queremos como país ingresar seriamente al crecimiento de nuestra industria y tener posibilidades reales e importantes para poder exportar nuestros servicios y productos, deberemos implementar mejores procesos y tener las certificaciones, pero para crecer de manera significativa hace falta una acción conjunta, o a través de un grupo de empresas o con apoyo del Estado, o de las universidades, porque las cifras de exportación de software nuestras son bajas comparadas con otros países.

Como resultado de la aplicación de un modelo de mejora con la norma ISO/ IEC 29110, los participantes coincidieron en señalar que sería conveniente que el registro de evidencia para determinar el cumplimiento de la norma sea automatizado y directo cuando los miembros del equipo de trabajo registren sus avances.

Lo expuesto confirma la disposición para implementar la mejora de procesos, adoptando una norma que les permita certificarse.

\section{Conclusiones y recomendaciones}

Con base en lo expuesto en el desarrollo del presente artículo, se presentan las siguientes conclusiones y recomendaciones:

\subsection{Conclusiones}

i. La revisión sistemática de literatura permitió identificar los modelos y normas más usados en las organizaciones de tipo mype, que les ha resultado útil en sus procesos.

ii. Las normas o modelos de referencias de procesos indican qué hacer pero no cómo. Esto último es atribuible a los métodos de desarrollo; por ello, en la propuesta de mejora de procesos se presenta una combinación de la norma ISO/IEC 29110 con algún método ágil, en principio en SCRUM.

iii. Las mypes productoras de software se encuentran dispuestas a adoptar un modelo de referencia de procesos, combinado con alguna práctica metodológica que permita garantizar la calidad del software elaborado.

iv. Las mypes productoras de software reconocen la importancia de disponer de un área de aseguramiento de calidad, independiente de la función de desarrollo de software. 
v. La propuesta de mejora de procesos incluye un modelo de evaluación vinculado a la norma ISO/IEC 29110 y a través del taller realizado se comprueba que es fácil de usar. Las mypes invitadas muestran una disposición para utilizarla, debido a las ventajas expuestas de la norma.

vi. Existen algunos elementos importantes que deben ser considerados en la implementación de un proyecto de mejora, tales como la definición de la misión, visión, objetivos estratégicos y análisis de riesgos que influyen directa y adecuadamente en la determinación del alcance del proyecto de mejora de procesos en las mypes productoras de software.

\subsection{Recomendaciones}

La validación cualitativa podría ser replicada teniendo en cuenta el mayor número de mypes que desarrollan sus actividades en Lima; y en provincias si se considera necesario.

Para una siguiente etapa de investigación, queda pendiente un proceso de validación cuantitativa del modelo propuesto en las empresas; es decir, el establecimiento de una línea base, y su comparación correspondiente con los resultados obtenidos con base en la implementación del modelo.

\section{Referencias}

Amable, M. (2015). Propuesta de un proceso de investigación cuantitativa. Aplicación en la caracterización de las mypes productoras de software. Interfases, 8, 71-92. Recuperado de https://revistas.ulima.edu.pe/index.php/Interfases/article/download/574/541

Amable, M., Millones, R., y Checa, R. (2015). Calidad de software en las mypes productoras de software en Lima. En Ugaz, J., Gonzales, S., y Torres, C. (Eds.), VII Congreso Internacional de Computación y Telecomunicaciones. Memoria COMTEL 2015 (133-139). Lima, Perú: Universidad Inca Garcilaso de la Vega, Fondo Editorial. Recuperado de http://www.comtel. pe/memoriacomtel/COMTEL2015.pdf

Barrios, M. (abril de 2010). Modelo de negocio. Asunción: Universidad Americana. Recuperado de http://marktur.travel/uploads/secciones_contenido/62/62-1323280539.pdf

Galván, S., Mora, M., O'Connor, R., Acosta, F., y Álvarez, F. (2015). A Compliance Analysis of Agile Methodologies with the ISO/IEC 29110 Project Management Process. Procedia Computer Science, 64, 188-195. DOI: 10.1016/j.procs.2015.08.480

García, J., Mitre, H., De Amescua, A., y Velasco, M. (2010). Integration of strategic management, process improvement and quantitative measurement for managing the competitiveness of 
software engineering organizations. Software Quality Journal, 18(3), 341-359. DOI: 10.1007/ s11219-010-9094-7

Garzás, J., Pino, F., Piattini, M., y Fernández, C. (2013). A maturity model for the Spanish software industry based on ISO standards. Computer Standards \& Interfaces, 35(6), 616-628. DOI: 10.1016/j.csi.2013.04.002

ISO / IEC. (2011). ISO/IEC TR 29110-3:2011. Software engineering - Lifecycle profiles for Very Small Entities (VSEs) - Part 3: Assessment guide.

Kitchenham, B., y Charters, S. (2007). Guidelines for performing Systematic Literature Reviews in Software Engineering. EBSE Technical Report EBSE-2007-01 (Vol. 2). Durham, Inglaterra. Recuperado de https://ees.elsevier.com/infsof/img/525444systematicreviewsguide.pdf

Mitre, H., De Amescua, A., García, J., y Velasco, P. (2014). Designing a Strategic Measurement Program for Software Engineering Organizations: Discovering Difficulties and Problems. Ingeniería, Investigación y Tecnología, 15(2), 253-269. DOI: 10.1016/S1405-7743(14)72215-2

Moreno, E., Sánchez, M., Colomo, R., y De Amescua, A. (2014). Towards Measuring the Impact of the ISO/IEC 29110 Standard: A Systematic Review. En Barafort, B., O'Connor, R., Poth, A. y Messnarz, R. (Eds.), Systems, Software and Services Process Improvement (1-12). Berlín, Alemania: Springer Berlin Heidelberg. DOI: 10.1007/978-3-662-43896-1_1

Niazi, M., Babar, M., y Verner, J. (2010). Software Process Improvement barriers: A cross-cultural comparison. En Information and Software Technology, 52(11), 1204-1216. DOI: 10.1016/j. infsof.2010.06.005

Osterwalder, A., y Pigneur, Y. (2011). Generación de modelos de negocio: Un manual para visionarios, revolucionarios y retadores. Barcelona, España: Deusto.

Trujillo, Y., Febles, A., y León, G. (2014). Modelo para valorar las organizaciones al iniciar la mejora de procesos de software. Ingeniare. Revista Chilena de Ingeniería, 22(3), 412-420. DOI: 10.4067/S0718-33052014000300011

Von Wangenheim, C., Rossa, J., Salviano, C., y Von Wangenheim, A. (2010). Systematic Literature Review of Software Process Capability/Maturity Models. En 10thInternationalSPICEConference on Software Process Improvement and Capability Determination, SPICE 2010. Recuperado de https://www.researchgate.net/profile/Jean_Hauck/publication/228738042_Systematic_ Literature_Review_of_Software_Process_CapabilityMaturity_Models/links/5542922e0cf 24107d39472e5/Systematic-Literature-Review-of-Software-Process-Capability-MaturityModels.pdf 
Wasson, C. (2015). System Engineering Analysis, Design, and Development: Concepts, Principles, and Practices (2. Ed.). Nueva York, Estados Unidos: John Wiley \& Sons, Inc.

Zarour, M., Abran, A., Desharnais, J., y Alarifi, A. (2015). An investigation into the best practices for the successful design and implementation of lightweight software process assessment methods: A systematic literature review. Journal of Systems and Software, 101, 180-192. DOI: 10.1016/j.jss.2014.11.04 
